\title{
A primary experience of conventional fractionated three-dimensional conformal partial breast irradiation for early-stage breast cancer
}

\author{
LINGXIA LIAO, GUANG HAN, YANPING LI, ZHAOHUA WANG, DONG LIU and ZHENGCHAO PI \\ Department of Radiotherapy, Hubei Cancer Hospital, Wuhan 430079, P.R. China
}

Received October 15, 2010; Accepted February 22, 2011

DOI: $10.3892 /$ etm.2011.223

\begin{abstract}
Recently, a number of clinical trials assessing partial breast irradiation (PBI), in particular accelerated partial breast irradiation, have been conducted in patients with early-stage breast cancer after breast-conserving surgery (BCS) in Europe and the US. By contrast, PBI is rarely performed in Eastern countries. In general, there are many physiological differences between Eastern and Western populations, and whether PBI is suitable for Eastern populations remains uncertain. Moreover, PBI is still in the stage of clinical research, and numerous questions have yet to be resolved. Thus, we designed a PBI trial to explore its feasibility and effectiveness for Eastern patients. According to the study criteria, 12 patients were enrolled between June 2003 and March 2007. Using three-dimensional conformal partial breast irradiation, they received a conventional fraction of $2 \mathrm{~Gy} /$ fraction/day, 5 fractions/week, DT60 Gy. According to follow-up data collected in August 2010, the rates of local recurrence and distant metastasis were $0 \%$. The most common adverse reactions included grade 2 radioactive erythema in $2(17 \%)$ cases and pigment deposition in $10(83 \%)$. The results revealed that the patients exhibited good local tumor control, with minor adverse reactions and satisfactory cosmetic results. More samples and long-term observations are required to further assess the validity and feasibility of PBI.
\end{abstract}

Correspondence to: Dr Guang Han, Department of Radiotherapy, Hubei Cancer Hospital, 116 Zhuodaoquan South Road, Hubei, Wuhan 430079, P.R. China

E-mail: hg7913@yahoo.com.cn

Abbreviations: PBI, partial breast irradiation; APBI, accelerated partial breast irradiation; BCS, breast-conserving surgery; CTV, clinical target volume; PTV, planning target volume; WBI, whole breast irradiation; EIC, extensive intraductal components; 3D-CRT, three-dimensional conformal radiotherapy; BED, equivalent biological dose

Key words: breast conservation therapy, three-dimensional conformal radiotherapy, conventional fractionation, partial breast irradiation

\section{Introduction}

Traditionally, patients undergoing breast-conserving surgery (BCS) receive standard whole breast irradiation (WBI) (1-3). However, patients with early-stage breast cancer are a heterogeneous group, with a variable risk and pattern of recurrence. Accordingly, alternative treatment options besides conventional WBI should be available. Based on findings that most recurrences after BCS for early breast cancer usually occur within or near the original tumor bed, partial breast irradiation (PBI) has become the focus of many cancer centers. Moreover, various centers have investigated the indications for BCS without radiotherapy (BCS only) (4,5). Thus, BCS and WBI, BCS and PBI or BCS only jointly constitute the individualized treatment strategy for early-stage breast cancer.

PBI is still in the clinical research stage. No consensus has been reached on numerous issues, such as indications, target area identification and fractionation methods. The aim of this study was to examine the use of PBI with a conventional fractionated three-dimensional conformal modality. Since the target volume was the only factor of this PBI protocol that varied from standard WBI, local tumor control was the focus and sole end point of this trial. Only once PBI has been determined to confer the same local tumor control benefits as WBI can this technique be examined in phase II trials.

\section{Materials and methods}

Patient eligibility. All patients had AJCC clinical stage I breast cancer, and the specific eligibility requirements were as follows: patients were $\geq 40$ years of age, had no history of prior malignancy and had unifocal breast cancer (single focus that could be encompassed by 1 tylectomy), $\mathrm{T} \leq 2 \mathrm{~cm}$ (T1), pN0, and pathological types that included invasive ductal carcinoma of grade I-II and non-lobular invasive carcinoma with no extensive intraductal components (EIC). In addition, patients were required to have surgical margins negative ( $\geq 5 \mathrm{~mm}$ ) for cancer.

Simulation, treatment planning and treatment. Treatment planning and delivery were performed with the patient in a supine position with a vacuum phantom fixed to expose the breasts. All patients underwent a CT breast simulation. The clinical breast borders and lumpectomy scar were marked with radiopaque catheters. CT images were acquired at 5-mm 


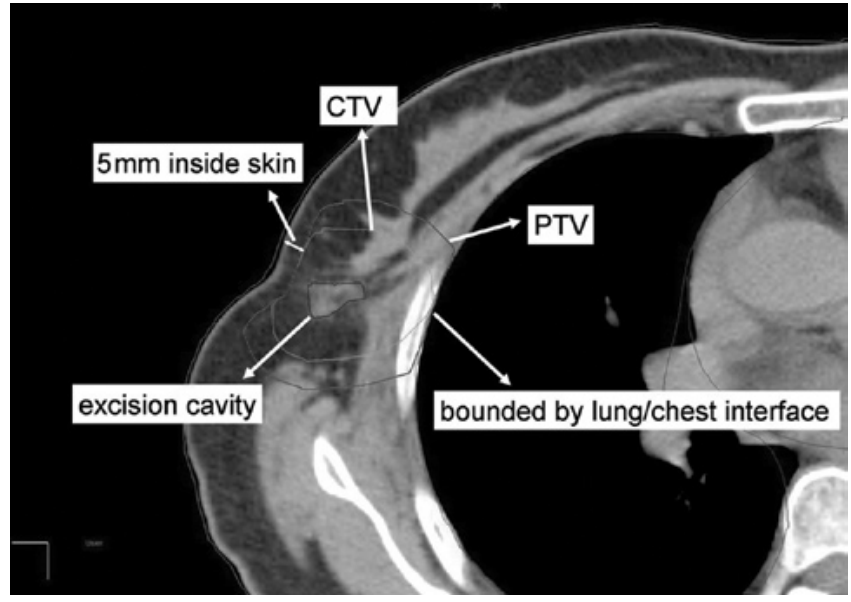

Figure 1. Clinical and planning target volumes. The clinical target volume (CTV) involved expanding the excision cavity volume by $15-20 \mathrm{~mm}$, which was limited to a 5-mm region from the skin surface and lung-chest wall interface. The planning target volume (PTV) included the CTV plus a $10-\mathrm{mm}$ margin and, similar to the CTV, was also limited to an area $5-\mathrm{mm}$ from the skin surface and lung-chest wall interface.

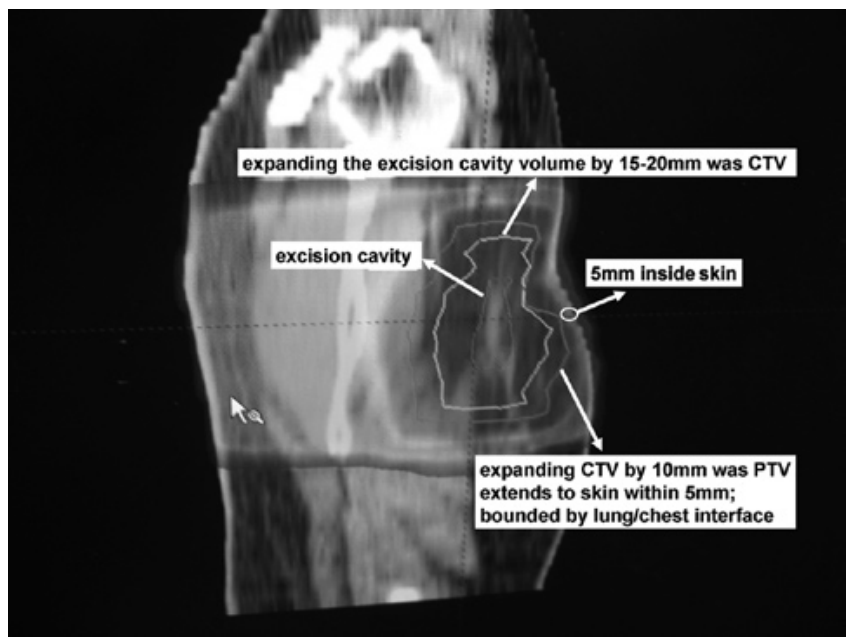

Figure 2. Sagittal section of the CTV and PTV as defined in Fig. 1.

intervals starting at or above the mandible and extending $2 \mathrm{~cm}$ below the inframammary fold to the costophrenic angle (including the entire lung). The lumpectomy cavity, ipsilateral breast, ipsilateral and contralateral lungs, and heart were contoured. The clinical target volume (CTV) was defined as uniform expansion of the excision cavity volume by $15-20 \mathrm{~mm}$ (Figs. 1 and 2). The post-operative scar, post-operative B ultrasonic and six surgical clips (Fig. 3) were used to determine the boundaries of the cavity volume. In order to ensure satisfactory cosmetic results and to reduce adverse reactions, the CTV was limited to an area $5 \mathrm{~mm}$ from the skin surface and lung-chest wall interface. The planning target volume (PTV) was designed to provide a margin around the CTV to compensate for the variability of treatment set-up and motion of the breast with breathing. A minimum 10-mm region surrounding the CTV was required (superior, inferior, medial and lateral dimension). Moreover, PTV was limited to exclude the region outside the patient and the first $5 \mathrm{~mm}$ of tissue under the skin and to also exclude (if applicable) the PTV expanded area

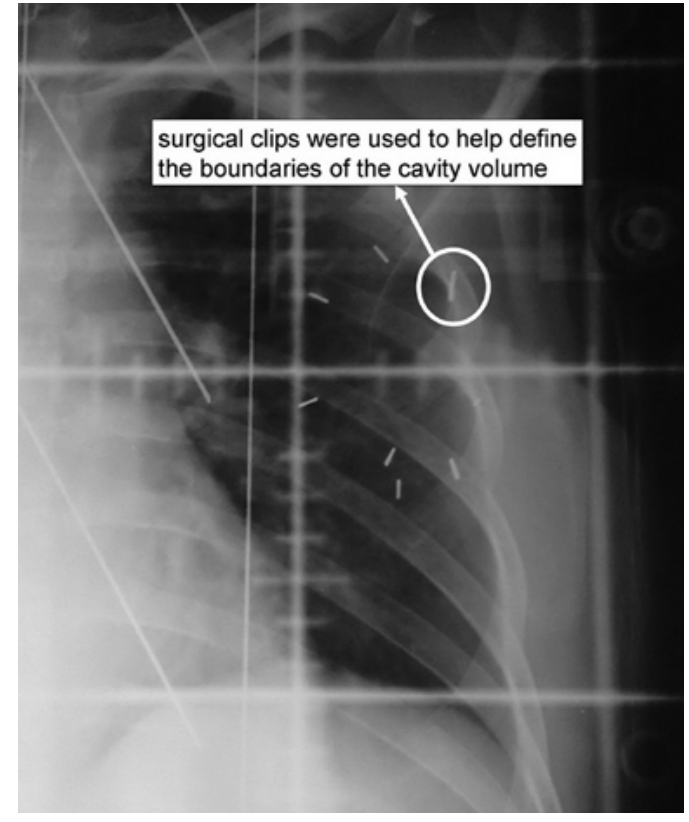

Figure 3. Clips were used to help define the boundaries of the cavity volume.

within the lung. Dose calculation was carried out using the organization's non-uniformity correction. Treatment planning was made by the Varian Eclipse TPS system. Three-to-five fields were arranged using 6-MV photons for lesions. Field arrangements were made at the discretion of the physician and determined by 3D treatment planning to produce the optimal conformal plan in accordance with volume definitions (described below). The treatment plan used for each patient was based on an analysis of the volumetric dose, including DVH analyses of the PTV and critical normal tissues.

When no chemotherapy was administered, it was recommended that radiotherapy start within 8 weeks of surgery. When chemotherapy was administered first, it was recommended that radiotherapy to start a minimum of 2 weeks after the last cycle of chemotherapy. A prescribed total dose of 60 Gy was administered, 2 Gy daily administered on 5 consecutive working days (from Monday to Friday).

\section{Normal tissue dose-limiting requirement}

Breast tissue dose limits. It was recommended that $\geq 50 \%$ of the ipsilateral whole breast receive $<60 \%$ of the prescribed dose, and that any point in the contralateral breast receive $<5 \%$ of the prescribed dose.

Lung tissue dose limits. It was recommended that $<15 \%$ of the ipsilateral lung receive $30 \%$ of the prescribed dose, and that $<15 \%$ of the contralateral lung receive $5 \%$ of the prescribed dose.

Heart tissue dose constraints. For right-sided lesions, it was recommended that $<5 \%$ of the heart receive $5 \%$ of the prescribed dose, while for left-sided lesions it was recommended that $<5 \%$ of the heart receive $40 \%$ of the prescribed dose.

Quality assurance evaluation. Ninety-five percent of the isodose surface covered $100 \%$ of the PTV. All critical normal tissue DVH limits were met. 
Statistical requirement. Reproducibility and safety were the early clinical trial primary end points. The optimal two-stage design by Simon was used (6). The p-value was taken as the true indicator of whether the final result was acceptable or marginally acceptable, and hence that the technique was reproducible. The statistical test hypothesis was: $\mathrm{H} 0, \mathrm{p} \leq \mathrm{p} 0$ $(=80 \%)$ vs. H1, $\mathrm{p} \geq \mathrm{p} 1 \quad(=95 \%)$, two types of errors, $\alpha=5 \%$, $\beta=10 \%$. Given these parameters, 19 eligible patients were required for the first stage of the two-stage design. However, in our experiments only 12 patients were eligible. When $\geq 2$ treatments from these 12 were scored as unacceptable, early discontinuation of the study was recommended; otherwise accrual of data was continued.

Follow-up study. The final date for the follow-up study was June 2010. Overall survival time was calculated as time from disease diagnosis to death or until the final date of the follow-up interval.

\section{Results}

The result of the follow-up study. All patients were followed up for at least 41 months. No case was lost and the follow-up rate was $100 \%$.

Patient characteristics. The pre-treatment characteristics for all eligible patients including the first 12 evaluable patients are shown in Table I.

Treatment efficacy and adverse reactions. All patients were followed up for at least 41 months, and presented a $0 \%$ rate of local recurrence and distant metastasis. Data regarding adverse reactions are presented in Table II.

\section{Discussion}

Individualized treatment for patients with early-stage breast cancer consists of BCS and irradiation (WBI or PBI) or BCS only, based on the fact that two subgroups of patients co-exist: those who are presumed to have occult disease and those who are free of tumor residue. However, it is not currently possible to identify these two patient subgroups.

To date, all trials regarding the omission of irradiation therapy, even in highly selected low-risk early breast cancer patients treated with BCS, have been unsuccessful (7-9). In addition, it has been consistently demonstrated that the local recurrence rate is significantly lower when adjuvant radiotherapy is administered to the mammary gland $(10,11)$. As a result, combined with the concept of the multicentric origin of breast cancer, WBI has become the standard procedure after BCS. However, many authors have questioned the necessity of WBI in early-stage breast cancer, since most of the local recurrences have been observed to occur near or close to the tumor bed, justifying the administration of PBI $(12,13)$. If PBI proves to be efficacious, it will offer the potential advantage of reduced treatment-related toxicity and improvement in the quality of life of patients by confining treatment to a limited volume of breast tissue adjacent to the lumpectomy cavity and by decreasing the volume of lung and heart that is irradiated as compared to WBI (14).
Table I. Pre-treatment characteristics for all eligible patients.

Patients $(n=12)$

\section{Age, years \\ Median \\ Range} 53

40-71

Surgery, n (\%)

Lumpectomy + axillary

lymph node dissection

Quadrantectomy + axillary

lymph node dissection

Axillary lymph node involvement

Median no.

Range

Histology, n (\%)

Invasive ductal

Ductal carcinoma in situ

Menopausal status, n (\%)

Premenopausal

7 (58)

Postmenopausal

Chemotherapy, n (\%)

None

Yes

Hormonal therapy, n (\%)

None

Yes

ER status, n (\%)

Positive

Negative

No test

PR status, n (\%)

Positive

Negative

No test

PS2 status, n (\%)

Positive

Negative

No test

HER-2 status, n (\%)

Positive

Negative

No test

A number of studies on PBI have been carried out in North America and Europe, and most clinical results are promising (15-17). Recently, a detailed systematic review analyzed almost all aspects of accelerated PBI (APBI), including the history of the patients and the rationale for the selection of the APBI, treatment techniques used, as well as the radiobiological aspects (18). In this review, the ipsilateral breast recurrence rates for most APBI cases were lower than $8 \%$ compared to patients receiving WBI. The most comprehensive study was from the William 
Table II. Adverse reactions related to radiotherapy in the studied patients $(n=12)$.

\begin{tabular}{lccc}
\hline Adverse reactions, $\mathrm{n}(\%)$ & Grade 1 & Grade 2 & Grade 3 \\
\hline Blood/bone marrow & $2(17)$ & 0 & 0 \\
Gastrointestinal & 0 & 0 & 0 \\
Lymphatic & $1(8)$ & 0 & 0 \\
Breast atrophy/tissue fibrosis & 0 & 0 & 0 \\
Pain & $1(8)$ & 0 & 0 \\
Pigmentation & $10(83)$ & $2(17)$ & 0 \\
Acute skin reaction & $8(67)$ & 0 & 0 \\
Breast hematoma & 0 & 0 & 0 \\
Pulmonary/lung fibrosis & 0 & 0 \\
\hline
\end{tabular}

Worst overall events: pigmentation 10 (83\%); acute skin reaction $2(17 \%)$.

Beaumont Hospital, USA, where 199 patients were enrolled from 1993. The inclusion criteria included patients $\geq 40$ years of age with ductal carcinoma only, $\mathrm{T} \leq 3 \mathrm{~cm}$, no EIC, pNO (prior to 1997 also $\mathrm{pN1}$ ) and negative surgical margins $\geq 2 \mathrm{~mm}$. The 5 -year actuarial local failure rate was only $1.2 \%$ after APBI with interstitial brachytherapy, as compared to WBI. We based our inclusion criteria for APBI on those of the William Beaumont Hospital (19), with certain adjustments: negative surgical margins were increased to $\geq 5 \mathrm{~mm}$ and only patients who placed extreme emphasis on the protection of normal tissues were included.

The only non-invasive technique for APBI is three-dimensional conformal radiotherapy (3D-CRT). The local failure rate for APBI by 3D-CRT is comparable to that of brachytherapy or other invasive techniques, such as mammosite or intraoperative radiation (20). 3D-CRT is more acceptable as it eliminates the need for an additional surgical procedure, has an improved dose homogeneity, and reduces the risk of fat necrosis. Furthermore, 3D-CRT has become common practice at most cancer centers, including those in developing countries, and does not require additional medical staff training, unlike brachytherapy. In the present study, 12 patients were treated with 3D-CRT; $95 \%$ of the isodose surface covered $100 \%$ of the PTV, the average area of the breast that received $115 \%$ of the prescribed dose was zero. The most common adverse reactions were grade 2 radioactive erythema in $2(17 \%)$ cases and pigment deposition in 10 $(83 \%)$. All patients had satisfactory cosmetic results.

In China, BCS is not a commonly opted for procedure compared to Western countries. In the West, younger women with a higher education level and a good socioeconomic status generally tend to undergo BCS. Some patients have no alternative besides BCS due to combined heart and/or lung morbidity. Almost all patients treated with BCS fulfill 5-6 weeks of conventional fractionated irradiation. In mainland China, patients who decide to undergo BCS are usually prepared for conventional fraction irradiation, but consider tumor control and improvement in the quality of life. Thus, our PBI trial design was focused on controlling the local tumor recurrence rate. In our opinion, the goal of any PBI trial is the comparability of the local tumor control of PBI to that of WBI. Although each of the APBI dose-fractionation schedules is determined by referring to the equivalent biological dose (BED) (21), in comparison with conventional fractionation, the BED is an indeterminate factor that may impact the results of an APBI trial. Therefore, a combination of conventional fractionation with 3D-CRT was used to ensure a logistically simpler and more practical method for PBI testing.

In our study, 12 patients complied with the conditions of the trial. The patients were followed up for more than 41 months, and no cases of recurrence or distant metastasis were noted. PBI and WBI appeared to demonstrate an equivalent local tumor control rate. In addition, the present study demonstrated the superior efficacy and cosmetic results of BPI, and the patients experienced few adverse reaction.

Due to the slow nature of local recurrences in breast cancer, a very long follow-up is required. This research is therefore still in its initial stages. Moreover, the number of cases was limited. Thus, further studies are necessary in order to reach an objective conclusion.

\section{Acknowledgements}

The authors wish to thank Ms. Xiaohong Wang, Ms. Jingya Wang and Mr. Fei Peng for their excellent technical assistance.

\section{References}

1. Fisher B, Anderson S, Bryant J, Margolese RG, Deutsch M, Fisher ER, Jeong JH and Wolmark N: Twenty year follow-up of a randomized trial comparing total mastectomy, lumpectomy and lumpectomy plus irradiation for the treatment of invasive breast cancer. N Engl J Med 347: 1233-1241, 2002.

2. Veronesi U, Cascinelli N, Mariani L, Greco M, Saccozzi R, Luini A, Aguilar M and Marubini E: Twenty year follow-up of a randomized study comparing breast conserving surgery with radical (Halstead) mastectomy for early breast cancer. $\mathrm{N}$ Engl J Med 347: 1227-1232, 2002.

3. Nold RJ, Beamer RL, Helmer SD and McBoyle MF: Factors influencing a women's choice to breast-conserving surgery versus modified radical mastectomy. Am J Surg 180: 413-418, 2000.

4. Fyles AW, McCready DR, Manchul LA, Trudeau ME, Merante P, Pintilie M, Weir LM and Olivotto IA: Tamoxifen with or without breast irradiation in women 50 years of age or older with early breast cancer. N Engl J Med 351: 963-970, 2004.

5. Malmström P, Holmberg L, Anderson H, Mattsson J, Jönsson PE, Tennvall-Nittby L, Balldin G, Lovén L, Svensson JH, Ingvar C, Möller T, Holmberg E, Wallgren A; Swedish Breast Cancer Group: Breast conservation surgery, with and without radiotherapy, in women with lymph node-negative breast cancer: a randomised clinical trial in a population with access to public mammography screening. Eur J Cancer 39: 1690-1697, 2003. 
6. Simon R: Optimal two-stage designs for phase II clinical trials. Control Clin Trials 10: 1-10, 1989.

7. Schnitt SJ, Hayman J, Gelman R, Eberlein TJ, Love SM, Mayzel K, Osteen RT, Nixon AJ, Pierce S, Connolly JL, Cohen P, Schneider L, Silver B, Recht A and Harris JR: A prospective study of conservative surgery alone in the treatment of selected patients with stage I breast cancer. Cancer 77: 1094-1100, 1996.

8. Liljegren G, Holmberg L, Adami HO, Westman G, Graffman S and Bergh J: Sector resection with or without postoperative radiotherapy for stage I breast cancer: five-year results of a randomized trial. J Natl Cancer Inst 86: 717-722, 1994.

9. Mannino $M$ and Yarnold J: Local relapse rates are falling after breast conserving surgery and systemic therapy for early breast cancer: can radiotherapy ever be safely withheld. Radiother Oncol 90: 14-22, 2008.

10. Clarke M: Meta-analyses of adjuvant therapies for women with early breast cancer: the Early Breast Cancer Trialists' Collaborative Group overview. Ann Oncol 17 (Suppl. 10): S59-S62, 2006.

11. Winzer KJ, Sauer R, Sauerbrei W, Schneller E, Jaeger W, Braun M, Dunst J, Liersch T, Zedelius M, Brunnert K, Guski H, Schmoor C and Schumacher M; German Breast Cancer Study Group: Radiation therapy after breast-conserving surgery; first result of a randomized clinical trial in patients with low risk of recurrence. Eur J Cancer 40: 998-1005, 2004.

12. Rosen PP, Fracchia AA, Urban JA, Schottenfeld D and Robbins GF: 'Residual' mammary carcinoma following simulated partial mastectomy. Cancer 35: 739-747, 1975.

13. Touboul E, Buffat L, Belkacémi Y, Lefranc JP, Uzan S, Lhuillier P, Faivre C, Huart J, Lotz JP, Antoine M, Pène F, Blondon J, Izrael V, Laugier A, Schlienger M and Housset M: Local recurrences and distant metastases after breast-conserving surgery and radiation therapy for early breast cancer. Int J Radiat Oncol Biol Phys 43: 25-38, 1999.
14. Park SS, Grills IS, Chen PY, Kestin LL, Ghilezan MI, Wallace M, Martinez AM and Vicini FA: Accelerated partial breast irradiation for pure ductal carcinoma in situ. Int J Radiat Oncol Biol Phys: Aug. 26, 2010 (Epub ahead of print).

15. Baglan KL, Sharpe MB, Jaffray D, Frazier RC, Fayad J, Kestin LL, Remouchamps V, Martinez AA, Wong $J$ and Vicini FA: Accelerated partial breast irradiation using 3D conformal radiation therapy. Int J Radiat Oncol Biol Phys 55: 302-311, 2003.

16. Vicini F, Winter K, Straube W, Wong J, Pass H, Rabinovitch R, Chafe S, Arthur D, Petersen I and McCormick B: A phase I/II trial to evaluate three-dimensional conformal radiation therapy confined to the region of the lumpectomy cavity for stage I/II breast carcinoma: initial report of feasibility and reproducibility of Radiation Therapy Oncology Group (RTOG) study 0319. Int J Radiat Oncol Biol Phys 63: 1531-1537, 2005.

17. Vicini FA, Kestin L, Chen P, Benitez P, Goldstein NS and Martinez A: Limited field radiation therapy in the management of early-stage breast cancer. J Natl Cancer Inst 95: 1205-1210, 2003.

18. Offersen BV, Overgaard M, Kroman $\mathrm{N}$ and Overgaard J: Accelerated partial breast irradiation as part of breast conserving therapy of early breast carcinoma: a systematic review. Radiother Oncol 90: 1-13, 2009.

19. Azria D and Hennequin C: Impact of radiotherapy modalities on local control and survival in adjuvant breast cancer treatment. Cancer Radiother 13: 434-445, 2009.

20. Bovi J, Qi XS, White J and Li XA: Comparison of three accelerated partial breast irradiation techniques: treatment effectiveness based upon biological models. Radiother Oncol 84: 226-232, 2007. 